\title{
Design of Smart Node and Routing Protocol Management in Wireless Sensor Networks
}

\author{
Huijuan Sun \\ Dept. of Information Engineering, Henan College of Finance \& Taxation, Zhengzhou 450046, China \\ huijuansunedu@163.com
}

Keywords: Wireless sensor network; Routing protocol; Sensor node topology; Simulation platform; Wireless communication

\begin{abstract}
Wireless sensor networks are interconnected by a large number of wireless sensor nodes, which are the extension of the sensor to the miniaturization, intelligence and wireless communication. This paper first analyzes the routing protocol of wireless sensor network, and discusses the data collection node management system based on wireless sensor. Then, this paper demonstrates the design and implementation of wireless sensor network from multiple applications. The experiments prove that the design method is effective.
\end{abstract}

\section{Introduction}

In recent years, the continuous progress of the microprocessor and the wireless communication technology produces to the local information processing and wireless communication devices are distributed, the small size, low price and low energy consumption of nodes called sensor nodes. Each sensor node can only be limited information processing, but a large number of nodes cooperate with each other can be a detailed observation of a given physical environment [1]. A large number of such sensor nodes form a wireless sensor network through the wireless communication technology. The wireless medium can be an infrared device or a radio, etc.. Different from traditional networks, wireless sensor networks rely on dense dispersion and mutual cooperation to complete their tasks.

In the use of wireless sensor networks, it is necessary to make reasonable use of energy is the extension of the use of the sensor network [2]. Safety management: wireless sensor network multi application in has the confidential nature of the project, so it is necessary to the security of application had certain requirements, mobility management: management of mobile is mainly responsible for the control node mobile, the maintenance of sink node routing. Network management: mainly for the network hardware and software systems for monitoring, control.

According to the requirements and characteristics of wireless sensor networks, there are many researches in this field, which can be divided into two parts: node level and network level. In node level of research content mainly includes sensor technology, low power consumption chip technology, wireless communication technology, the research content of network level mainly include low energy consumption routing technology, low energy consumption of the MAC protocol, collaborative positioning technology, clock synchronization technology, data fusion technology.

\section{Intelligent Node and Routing Protocol Management for Wireless Sensor Networks}

Wireless sensor network is a new information acquisition and processing technology, it is a new type of wireless sensor networks. Sensor nodes in wireless sensor networks complete some data acquisition work, the nodes send data to the network through the sensor network, and eventually received by the specific application. The sensor node integrates sensors, data processing unit and communication module, and the network is formed by self organizing method.

The actual relationship between the output and the input of the sensor is off to the degree of fitting a straight line. It is defined as the ratio of the maximum deviation value between the actual characteristic curve and the fitting line is to the full scale output value in the full scale range. 
Sensitivity: sensitivity is an important indicator of the static characteristics of the sensor [3]. It is defined as the ratio of the increment of the output and the increment of the corresponding input increment.

Node is the information acquisition and based on OPNET wireless sensor network QoS routing and traffic modeling and Simulation of an also acted as the routing information, collected data through multi hop routing arrive at the sink node [4]. The sink node, as a special node, can communicate with the monitoring center through Internet, mobile communication network, satellite and so on. It can also take advantage of the UAV flight over the network, through the sink node to collect data.

Sensor networks are often deployed in the environment is very harsh conditions, the open work environment the often exposed to wind and rain, or by animal do not intend to damage. In order not to affect the normal operation of the sensor network, it is necessary to require the sensor network has a strong reliability. For the reliability of another kind of understanding is that the sensor nodes to collect monitoring data must be true and reliable, otherwise, it will not be for the working environment provide effective scientific data, guidance on the work environment also disappeared, as is shown by equation (1).

$$
\gamma(m+1)=\Phi_{w}(m) x(m, M)+\bar{w}_{\gamma}(m)
$$

The network layer routing protocol of wireless sensor network for the whole network balanced energy consumption, and is generally used to data path for the center to establish the data source to the sink node. The main research contents of this layer include topology control, time synchronization and location [5]. Topology control for wireless sensor networks can be divided into two categories, one is the node power control is a kind of hierarchical topology control.

Active route discovery strategy is similar to the traditional routing protocols, nodes periodically broadcast routing information packet exchange routing information, take the initiative to find a route, and all the nodes in the network must keep of all nodes on the route, the active routing protocol, is maintained between all the nodes in the network is usually the source node and the destination node routing table, regardless of the route if necessary. Active routing protocols usually include routing and broadcasting "two neighbor" detection process. The formula used in the method is as follows [6].

$$
\frac{\partial F}{\partial a}=-2 \sum_{i=0}^{n}\left(y_{i}-a x_{i}-b\right)=0
$$

GPEAR routing mechanism, the receiving sensitivity under certain circumstances, the relationship between the transmitting power and receiving radius is $\mathrm{P}$, and $\mathrm{R}$ is proportional to $\mathrm{R} 2 \sim \mathrm{R} 5$, or $\mathrm{P}$ may be far greater than $\mathrm{R} 2$. If communication is considered in the inter node communication distance, appropriate adjustments to the transmission power, instead of using the same transmit power (so only the maximum communication distance to launch), equation (3) can greatly reduce the communication energy consumption and prolong the lifetime of the entire network, reduce each number according to the communication cost of the package [7].

$$
P_{i}(k \mid k-1)=\Phi_{i} P_{o i}(k-1) \Phi_{i}+Q_{i}
$$

Wireless sensor network architecture in the third part, the application software. This part includes all according to the user's existing development software code, the code is currently mostly using $\mathrm{C}$ language development, can to interface and API, call software protocol stack, in a wireless sensor network technology, we believe that use 802.15.4 international standard and ZigBee technology, as we design wireless sensor system started.

Step1: The characteristics of the wireless sensor network with large scale, high density and narrow band width makes its internal communication interference. So WSN needs to achieve a simple, strong anti-interference ability, low power consumption and low cost of modulation and spreading mechanism; 
Step2: The wireless communication module as data transmission channel system is important, to realize data transmission and network function between the acquisition node and sink node, collection node and acquisition node. In this design, the combined use of CC2531 and CC2591 wireless communication module [8]. CC2591 TI for wireless applications of low power and low voltage, the highest integration $2.4 \mathrm{GHz} \mathrm{RF}$ front end. It integrates the power amplifier (which can increase the output power of +22 , low noise amplifier $(\mathrm{dBm})$ receiver can be improved sensitivity $+6 \mathrm{~dB})$, (balun), balance converter switches, inductors and RF matching network is defined as:

$$
k_{N}(x)=(2 \pi)^{-d / 2} \exp \left(-\frac{1}{2} x^{2}\right)
$$

Step3: The sensor provides matching target state, attribute information. On each sensor transmits to all the relevant information of data fusion; identified in a sensor management cycle each sensor agent required to complete the tasks of the system and their global performance index; performance index of system task monitoring, in order to confirm the required performance indicators are reached;

Step4: RSSI, received signal strength indicator) refers to the wireless signal intensity received by node. In localization is based on received signal strength indicator in RSSI.

Step5: OPNET mainly includes the following four products: verification of hardware design of OXC network planning and design tool IT Decision Gure, Modeler, R \& D network simulation platform of wireless mobile network simulation and network simulation in Modeler/Radio:

$$
\frac{\partial^{2} \phi}{\partial x^{2}}+\frac{\partial^{2} \phi}{\partial y^{2}}+f(x, y) \phi=q(x, y)
$$

Step6: Rumor routing mechanism introduced the query message unicast random forwarding, overcome by using flooding to establish forwarding path overhead is too big.

Using AD990 is as a sensor. AD590 is the current output ends of temperature sensor which is made using the PN node and the temperature of the forward current. This device is in the measured temperature, the equivalent of a constant current source. This device has good linearity and interchangeability, high measuring precision, and has the characteristics of eliminating power fluctuation.

In the selection of the sensor nodes for environment monitoring, we must choose the small size, high precision, and long life cycle as the monitor node. As the sensor nodes may reduce the volume, and the use of heterogeneous sensor nodes, in order to adapt to the complexity of the environment sensitivity and monitoring environment for foreign equipment. High precision sensors are more conducive to accurately obtain the environment parameters [9]. The starting time is too long will consume a lot of energy; persistence is not conducive to the work of sensor nodes. Therefore, when the design needs are to choose the sensor short start-up time to save energy.

\section{Design and Implementation of Wireless Sensor Network Topology}

Although the wireless sensor networks and wireless ad hoc networks are similar, but there are also great differences. Sensor network is the integration of monitoring and control and wireless communication network system, the number of nodes larger (thousands or tens of thousands), nodes are distributed more intensive; due to environmental impact and energy depletion, nodes are more prone to failure; environmental interference and node failures could easily lead to changes in the network topology.

This paper adopted the method of monitoring and management program is provided by XServe standard data interface to develop custom wireless sensor network. Interface with XML XServe output as a data source to connect to the XServe server through Socket middleware, XServe middleware server can be configured to XML data TcP / IP protocol based on current output.

CSMA/ collision are difficult to use in the application of wireless sensor network detection system. But can choose an alternative, CSMA collision avoidance (CA) is an effective method for collision 
control. However, CSMA-CA will increase the additional traffic greatly to the network. If there is no direct collision control method, the data link layer error control method should be used to ensure certain transmission success rate. In fact, the cyclic redundancy check general error detection technique with a response handshake signal is very effective in sensor networks. The data link layer response (node to node) and network layer response (end-to-end) flexible combination can meet the performance requirements of the transmission success rate, and achieve the desired level of power consumption, as is shown by equation(6) [10].

$$
X^{\prime}=W(X, P)=\left(\begin{array}{ccc}
a_{1} & a_{2} & d_{1} \\
a_{3} & a_{4} & d_{2} \\
0 & 0 & 1
\end{array}\right)\left(\begin{array}{l}
x \\
y \\
1
\end{array}\right)
$$

The advantages of hardware and software localization engine WSN positioning method is compared with high speed and high precision, no processor time. The location engine main features are as follows: location estimation algorithm needs 3 8 reference node; localization estimation to $0.5 \mathrm{~m}$. Computing nodes position takes less than 40 microseconds; range free localization for $64 \mathrm{M}$; positioning deviation less than $3 \mathrm{~m}$; positioning engine using distributed computing method, the method using known reference node RSSI positioning information. In the distributed node location calculation can avoid a large number of network transmission and communication delay calculation method of concentrated problems.

\section{Experiments and Analysis}

Efficient utilization of network energy is a significant feature of sensor network routing protocol, some routing protocols proposed earlier often only consider the factor of energy. In order to emphasize the importance of efficient use of energy, so they will be divided into energy aware routing protocol. Energy aware routing protocol from the data transmission energy consumption, and discuss the optimal energy consumption path and the maximum network lifetime problem.

For the design and management of wireless sensor network, generally divided into three stages: the first stage for the design stage, including the design of network topology, protocol design and configuration and network equipment design and selection; the second stage for release phase, design of network can have certain properties, such as throughput, response time, and so on; the third stage for the actual operation of fault diagnosis, troubleshooting, and upgrade optimization:

(1) The remote control task is mainly used for monitoring task coordination in sensor networks. This work is mainly carried out in the remote data server, by remote control coordination after work will coordinate the results after transfer to the entire network;

(2) When the need for the same destination to start a new route discovery, it must increase its own serial number. This will prevent and prior to the establishment of the source node to the reverse path conflict, as is shown by equation (7).

$$
\lambda_{1}(k)=\left\|\frac{\hat{X}_{o 1}(k)-\hat{X}_{1}(k)}{\hat{X}(k)-\hat{X}_{1}(k)}\right\|
$$

(3) In Xserve, open the case, you can read the TCP data control data interception down again by the XML parser developed.

Although OPNFT is powerful simulation platform and the price is expensive. G1oMoSim of the Free is University. However, access to the software is still not easy. In addition, the main advantage of this software is simulation of large scale network (multiple nodes in more than 1000), and that is the focus of this paper is small and medium-sized network simulation (the number of nodes in a 500 below), so there is no use. NS2 is a free, open, easy to obtain, especially suitable for use in scientific 
research. This paper mainly uses the NS2 of WSN research, and NS-2 plus CMU wireless module, as shown in Fig. 1.

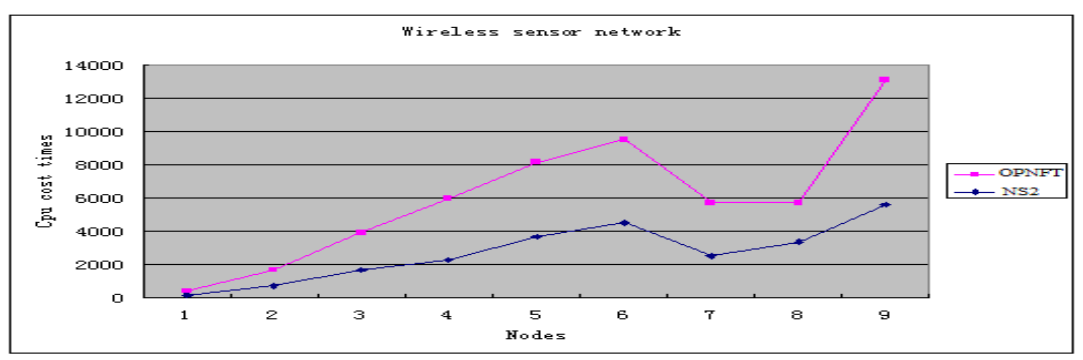

Figure 1. Comparison of routing protocol management in wireless sensor networks by OPNFT with NS2

From the experiment in Fig. 1 we can see that when reaching the steady state, the background traffic conditions, the response time of NS2 download increased nearly 5 seconds. Here through the display background traffic situations, link utilization and throughput than high background traffic. The performance parameters of the network are also other can view through the collection of statistics and operation simulation.

\section{Summary}

In a dynamic network, sensor nodes are generally placed in the movable object, such as a vehicle or by monitoring the animal, it will move along with the object. Due to the application of wireless sensor network has broad prospects, it has become a research hotspot at home and abroad. As a developing technology, there are still many problems need to be solved in wireless sensor network.

\section{References}

[1] K.Akkaya and M.Younis. A Survey on Routing Protocols for Wireless Sensor Networks. Elsevier Ad Hoc Network Journal, vol.3.pp.325-349,2005

[2] AkyildizF, SuW, Sankara subramanianmY, CayirciE. Wireless sensor networks:Asurvey. Computer Networks, 2002,38(4):3932422.

[3] M. Chu , H. Haussecker , F. Zhao. Scalable Information-Driven Sensor Querying and Routing for ad hoc Heterogeneous Sensor Networks. The International Journal of High Performance Computing Applications , 2002,16 (3).

[4] Chong C Y, Kumar S P. Sensor networks: evolution, opportunities and challenges.Proc. of the IEEE, 2003, 91(8): 1247-1256.

[5] J.Rethna Virgil Jeny, Dr.E.Babu Raj, "Analysis of Energy Efficient Cluster Based Routing Protocol for Wireless Sensor Networks", JCIT, Vol. 10, No. 2, pp. 09 16, 2015.

[6] C. Intanagonwiwat, R. Govindan, D. Estrin, J. Heidemann, and F. Silva, "Directed diffusion for wireless sensor networking," IEEE/ACM Trans. Netw., vol. 11, no. 1, pp. 2-16, 2003.

[7] Sardjoeni Moedjiono, Aries Kusdaryono, Teddy Mantoro, "Energy Efficient Base Station Assisted Cluster Routing Protocol in Wireless Sensor Networks (BCRP)", IJACT, Vol. 6, No. 4, pp. $01 \sim 13,2014$.

[8] Zhe Qu, Tao Du, "An Energy Aware Clustering Routing Algorithm for No Identity Wireless Sensor Networks", AISS, Vol. 6, No. 3, pp. 64 75, 2014.

[9] F. Ye, H. Luo, J. Cheng, S. Lu, and L. Zhang, "A two-tier data dissemination model for large-scale wireless sensor networks," in Proceedings of the 8th annual international conference on Mobile computing and networking. ACM Press, 2002, pp. 148-159.. 
[10] A. Sinha, A Chandrakasan. Dynamic power management in wireless sensor networks. Design \& Test of Computes, 2001, 18(2): 62-74. 